\section{Scleral buckling combined with vitrectomy for the management of rhegmatogenous retinal detachment associated with inferior retinal breaks}

P Alexander, A Ang, A Poulson and MP Snead
Department of

Ophthalmology,

Vitreoretinal Service,

Addenbrooke's Hospital,

Cambridge, UK

Correspondence: MP Snead,

Department of

Ophthalmology,

Vitreoretinal Service,

Addenbrooke's Hospital,

Hills Road,

Cambridge CB2 2QQ, UK

Tel: + 441223 216701;

Fax: + 441223217968

E-mail: mps34@

cam.ac.uk

Received: 13 June 2006 Accepted: 7 July 2006

Published online:

1 September 2006

No proprietary interests or research funding

\begin{abstract}
Aim The use of adjunctive scleral buckling during vitrectomy for retinal detachment associated with inferior breaks has been the recent focus of some debate. It has been suggested that any benefit might be outweighed by potential complications associated with buckling surgery. The purpose of this study was to compare the success rate of vitrectomy, gas, and supplementary scleral buckling with the results of two recently published case series suggesting that acceptable results in inferior break detachments can be achieved with vitrectomy and gas alone. Methods A retrospective analysis of 60 consecutive patients with inferior break retinal detachments was conducted. All patients underwent vitrectomy, gas, and scleral buckling. In all cases, fellow eyes were examined under anaesthesia for retinal breaks if posterior vitreous detachment (PVD) had occurred at the time of presentation.

Demographics, preoperative, and postoperative complications and outcome were recorded.

Results Primary retinal attachment at 3 months was achieved in $95 \%$ of patients. This exceeds success rates of published data of patients who underwent vitrectomy and gas without buckling (81-89\%). In patients with PVD in the fellow eye, 53\% had horseshoe tears and all were treated with cryopexy. No sight-threatening complications occurred, but five patients developed minor buckle-related complications postoperatively.
\end{abstract}

Conclusion Supplementary scleral buckling is a safe procedure that improves and enhances primary success rates in inferior break detachments over vitrectomy and gas without buckling. There is a high rate of PVDrelated breaks requiring treatment in the fellow eyes.

Eye (2008) 22, 200-203; doi:10.1038/sj.eye.6702555; published online 1 September 2006

Keywords: retinal detachment; scleral buckling; vitrectomy

\section{Introduction}

Over the past two decades, pars plana vitrectomy with gas tamponade has become increasingly popular in the primary repair of rhegmatogenous retinal detachment. Although approximately half of all cases can be safely managed with scleral buckling, without the need for vitrectomy, ${ }^{1}$ the latter is preferred in cases of vitreous opacity, bullous detachments, or multiple, posterior breaks. However, as intraocular gas tamponade is unlikely to provide effective tamponade for inferior breaks, such patients often undergo combined vitrectomy and scleral buckling procedures. Recently, however, two groups have called into question the need for supplementary scleral buckling in such cases, suggesting that vitrectomy alone can achieve acceptable success rates and that the theoretical advantages of buckling are outweighed by their potential 
complications. In a retrospective analysis, Sharma et $a l^{2}$ compared outcomes of vitrectomy surgery in a group of patients with inferior break retinal detachments with a group of patients with superior breaks, and could not demonstrate a statistically significant difference in their series. Wickham et $\mathrm{al}^{3}$ conducted a retrospective casenote review of inferior break retinal detachments, and noted that approximately half of the patients were treated with vitrectomy alone, whereas the other half had combined vitrectomy and buckle surgery. No significant difference in outcome was demonstrated, but the authors admit that the latter group may have had undocumented preoperative proliferative vitreoretinopathy (PVR) which determined the choice of surgical procedure, thereby precluding comparison between the groups.

It has been argued that by avoiding supplementary scleral buckling, the associated complications such as refractive changes, ${ }^{4}$ diplopia, ${ }^{5,6}$ explant extrusion, or infection ${ }^{7}$ are prevented. Placement of a scleral buckle on to a hypotonous vitrectomised eye increases the risk of choroidal haemorrhage. ${ }^{8}$

In this paper, we review the results of our patients with inferior break retinal detachments who were all treated with vitrectomy, gas tamponade, and supplementary scleral buckling. We compare these results with previously published data from groups who have not performed supplementary buckling.

\section{Methods}

A retrospective analysis of patients managed by the vitreoretinal service at Addenbrooke's Hospital was conducted. An electronic database was used to compile an unselected consecutive series of all patients under the care of a single surgical team who underwent pars plana vitrectomy, intraocular gas tamponade, and scleral buckling for retinal detachment associated with inferior horseshoe tears between January 1999 and January 2005. Exclusion criteria included previous retinal detachment surgery, PVR of Grade C or greater, round hole detachments (which can be safely managed with buckling alone ${ }^{9}$ ), giant retinal tears, and retinoschisis. Data collected on each patient included demographics, ocular co-morbidity, and type and distribution of retinal breaks. All patients had follow-up for at least 3 months.

Preoperative assessment by the vitreoretinal consultant included indirect ophthalmoscopy and slit-lamp biomicroscopy of the vitreous and retina of both eyes. All patients underwent scleral buckling (with either a silicone sponge or encircling 240 silicone band) followed by two-port vitrectomy under indirect ophthalmoscopic control. Retinal breaks were treated with monitored trans-scleral cryotherapy, and intraocular tamponade was achieved with either $\mathrm{SF}_{6}$ or $\mathrm{C}_{3} \mathrm{~F}_{8}$.
The fellow eye was also examined at the time of presentation. If posterior vitreous detachment (PVD) was present, the fellow eye was also examined under anaesthesia, and any identified retinal breaks were treated with trans-scleral cryotherapy. Perfluorocarbon liquids were not necessary as adjuncts to surgery in any case. All surgical procedures were conducted under general anaesthetic. The primary outcome measure was the retinal reattachment rate at 3 months following surgery.

\section{Results}

A total of 60 patients were included in the study, with a mean age of 60.1 years, and a male to female ratio of $2: 1$. Mean follow-up was 17 months (range 3 months to 4 years). Right and left eyes were equally affected (31 and 29 eyes, respectively). The intraocular gas used was $\mathrm{SF}_{6}$ in 50 patients, and $\mathrm{C}_{3} \mathrm{~F}_{8}$ in 10 patients.

Primary retinal reattachment was achieved in 57 of 60 patients (95\%). Three patients developed retinal redetachment. Two of these developed new inferior breaks, whereas the other had a tractional inferior retinal break posterior to the original break. Thirty-six patients were diagnosed with PVD in the fellow eye preoperatively. Of the 36 fellow eyes, five patients had had previous retinal detachments. Of the remaining 31 eyes, retinal breaks were identified in 14 eyes. Therefore, bilaterality of retinal breaks was present in 19 of 36 patients (53\%) with PVD in the fellow eye. A further three patients developed retinal breaks at the time of PVD in the fellow eye during the follow-up period.

Complications associated with scleral buckling were noted in five patients, but none of these was sight threatening. One patient had a small inferior choroidal haemorrhage noted on the first postoperative day, which spontaneously resolved, with a final visual acuity of $6 / 5$. This patient later underwent removal of the buckle under local anaesthetic because of discomfort. Two further patients also underwent buckle removal because of either discomfort or cosmesis. Two patients had postoperative diplopia that resolved spontaneously and required no further surgical intervention.

\section{Discussion}

This study showed a success rate of $95 \%$ in patients undergoing vitrectomy, gas tamponade, and supplementary scleral buckling for rhegmatogenous retinal detachment with inferior horseshoe tears. The decision to use a combined approach with vitrectomy and supplementary scleral buckle was taken in the presence of inferior retinal breaks. Whereas intraocular gas tamponade can support the nasal or temporal retina 


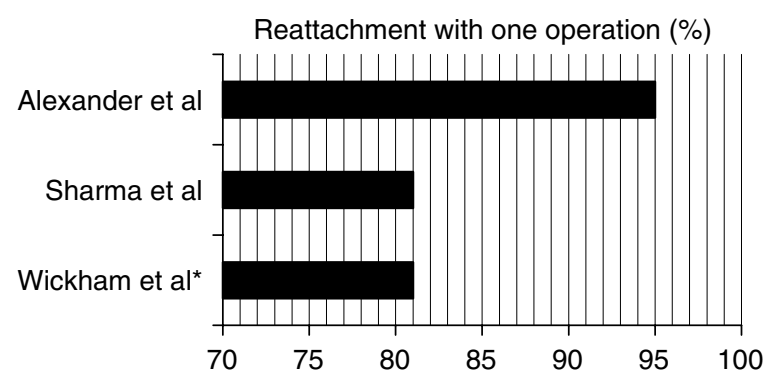

Figure 1 *Wickham et al's retrospective study of inferior break detachments showed an overall primary success rate of $81.4 \%$ (89\% for vitrectomy and gas alone, $73 \%$ for vitrectomy, gas, and buckle).

with good posturing, supplementary scleral buckling is traditionally regarded as necessary for retinal detachments with inferior breaks. ${ }^{10}$

Placement of a supplementary scleral buckle is technically demanding, prolongs the operating time, and can be associated with exposure, extrusion, ${ }^{7}$ or intrusion of the implant, refractive change ${ }^{4}$ diplopia, ${ }^{6}$ choroidal haemorrhage, and possibly anterior segment ischaemia. ${ }^{11}$ However, these risks may be justified if superior outcomes are achieved. Our success rate exceeded that of previous groups who have treated inferior break detachments with vitrectomy and gas alone (Figure 1). Wickham $e^{a} \mathrm{l}^{3}$ achieved an overall success rate of $81 \%$ in patients with inferior detachments. Their own retrospective analysis failed to demonstrate any difference between a group of patients who underwent vitrectomy, gas, and buckling, and a second group of patients with inferior detachments who underwent vitrectomy and gas only. They concluded that vitrectomy and gas without buckling has a role in the treatment of inferior break detachments. The retrospective nature of the study brings its own flaws. As McLeod ${ }^{12}$ points out in his editorial, the decision to buckle might have been based on some manifestations of early PVR, which would therefore have rendered anatomical failure more likely. Postoperatively, Wickham et $a l^{3}$ found that the patients who received buckles were more likely to develop PVR, and the authors admit that even though PVR was used as an exclusion criterion, some patients in the group may have had early undocumented PVR preoperatively.

Following a pilot study of eight patients by Tanner et $a l^{13}$ Sharma et $a l^{2}$ also conducted a retrospective case analysis of patients who underwent vitrectomy and gas, without buckling, for inferior break detachments and achieved a primary success rate of $81.3 \%$. The authors compared the results of this group with patients who had vitrectomy and gas for retinal detachments without inferior breaks, and could not find a significant difference in outcome.
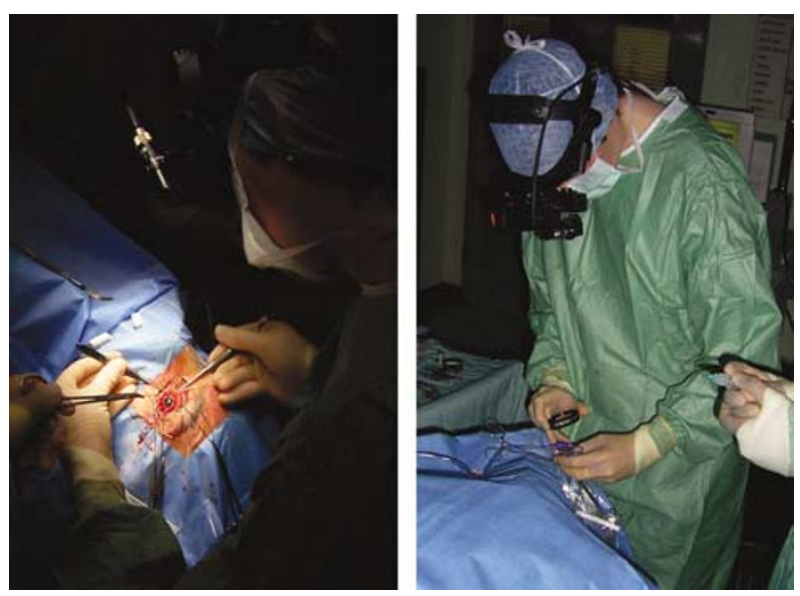

Figure 2 Use of the binocular indirect ophthalmoscope throughout the procedure allows seamless transition between buckling (left) and vitrectomy (right).

In their retrospective series of 512 patients who underwent primary vitrectomy for retinal detachment, Heimann et $\mathrm{al}^{14}$ found a significantly higher rate of redetachment in patients with inferior detachment. They suggest logically that their use of relatively short-acting tamponades are probably not sufficient to support the inferior retina with supine posturing. They also comment that inferior detachments tend to be present for longer before patients seek clinical advice, and that preoperative intravitreal fibrocellular proliferations may therefore be more common than in superior detachments, but this is unproven. $^{15}$

All of our patients underwent a two-port vitrectomy with indirect ophthalmoscopy. This has a number of advantages over microscope-based wide angle viewing systems. It allows seamless transition between buckling and vitrectomy (Figure 2), and the operation time is therefore not prolonged unnecessarily, unlike with microscope based systems that can be cumbersome and inflexible during a combined vitrectomy and buckling procedure. The average operation time in our unit for a combined procedure using indirect vitrectomy is $45 \mathrm{~min}$. Vitrectomy under indirect ophthalmoscopic control allows the surgeon to move around the patient and gives greater flexibility in access and visualisation. In addition, only two ports are required so the risks of port-related complications are minimised.

The absence of sight-threatening complications attests to the safety of the scleral buckling technique. There is a risk of choroidal haemorrhage during placement of the scleral buckle on a hypotonous eye following vitrectomy. Our practice is to localise and place the buckle before commencing vitrectomy, thereby reducing the risk of choroidal haemorrhage or scleral perforation.

Our study also demonstrated a high prevalence of bilaterality of retinal breaks in this subgroup of patients. 
In the presence of PVD in the fellow eye, more than half of the patients either had a previous retinal detachment or had retinal breaks that were identified at the time of surgery in the fellow eye. All retinal detachment surgery in our unit is performed under general anaesthetic, which facilitates careful examination with scleral indentation.

In conclusion, our results support the use of supplementary scleral buckling for patients undergoing vitrectomy and gas for primary rhegmatogenous retinal detachment with inferior breaks, particularly in situations where there are breaks in both inferior quadrants. The procedure appears quick and safe. Primary success rates are higher than those reported for vitrectomy and gas alone, and no sight-threatening complications occurred in our series. The importance of examining the fellow eye for retinal breaks because of the high rate of bilaterality found in our patients is emphasised, and that examination under general anaesthetic may make this easier and more effective.

\section{References}

1 Alexander P, Prasad R, Ang A, Poulson AV, Snead MP. Prevention and control of PVR using a selective two stage approach. (in submission).

2 Sharma A, Grigoropoulos V, Williamson TH. Management of primary rhegmatogenous retinal detachment with inferior breaks. Br J Ophthalmol 2004; 88: 1372-1375.

3 Wickham L, Connor M, Aylward GW. Vitrectomy and gas for inferior break retinal detachments: are the results comparable to vitrectomy, gas and scleral buckle? Br J Ophthalmol 2004; 88: 1376-1379.
4 Goel R, Crewdson J, Chignell AH. Astigmatism following retinal detachment surgery. Br J Ophthalmol 1983; 67: 327-329.

5 Arruga A. Motility disturbances induced by operations for retinal detachment. Mod Probl Ophthalmol 1977; 18: 408-414.

6 Fison PN, Chignell AH. Diplopia after retinal detachment surgery. Br J Ophthalmol 1987; 71: 521-525.

7 Flindall RJ, Norton EW, Curtin VT, Gass JD. Reduction of extrusion and infection following episcleral silicone implants and cryopexy in retinal detachment surgery. Am J Ophthalmol 1971; 71: 835-837.

8 Tabandeh H, Sullivan PM, Smahliuk P, Flynn Jr HW, Schiffman J. Suprachoroidal haemorrhage during pars plana vitrectomy. Risk factors and outcomes. Ophthalmology 1999; 106: 1811-1815.

9 Ung T, Comer MB, Ang AJ, Sheard R, Lee C, Poulson AV et al. Clinical features and surgical management of retinal detachment secondary to round retinal holes. Eye 2005; 19: 665-669.

10 Gartry DS, Chignell AH, Franks WA, Wong D. Pars plana vitrectomy for the treatment of rhegmatogenous retinal detachmenty uncomplicated by advanced proliferative vitreoretinopathy. Br J Ophthalmol 1993; 77: 199-203.

11 Kwartz J, Charles S, McCormack P, Jackson A, Lavin M. Anterior segment ischaemia following segmental scleral buckling. Br J Ophthalmol 1994; 78: 409-410.

12 McLeod D. Is it time to call time on the scleral buckle? Br J Ophthalmol 2004; 88: 1357-1359.

13 Tanner V, Minihan M, Williamson T. Management of inferior retinal breaks during pars plana vitrectomy for retinal detachment. Br J Ophthalmol 2001; 85: 480-482.

14 Heimann H, Zou X, Jandeck C, Kellner U, Bechrakis NE, Kreusel KM et al. Primary vitrectomy for rhegmatogenous retinal detachment: an analysis of 512 cases. Graefe's Arch Clin Exp Ophthalmol 2006; 244: 69-78.

15 Afrashi F, Erakgun T, Akkin C, Kaskakaloglu M, Mentes J. Conventional buckling surgery or primary vitrectomy with silicone oil tamponade in rhegmatogenous retinal detachment with multiple breaks. Graefe Arch Clin Exp Ophthalmol 2004; 242: 295-300. 